\title{
SOME OPTICAL AND EPR PROPERTIES OF STRAIN-FREE GaN CRYSTALS OBTAINED BY AMMONO METHOD*
}

\author{
R. DWiliński, J.M. Baranowski, M. KamińsKa \\ Institute of Experimental Physics, Warsaw University \\ Hoża 69, 00-681 Warsaw, Poland
}

\section{R. DORADZIŃSKI}

Institute of Theoretical Physics, Warsaw University, Hoża 69, 00-681 Warsaw, Poland

\section{J. GarCZyŃski, L. SierzPutowski}

Dept. of Chemistry, Warsaw Univ. of Technology

Noakowskiego 4, 00-661 Warsaw, Poland

\section{and M. PalczewsKa}

Institute of Electronic Materials Technology, Wólczyńska 133, 01-919 Warsaw, Poland

AMMONO GaN is grown spontaneously from ammonia solution in form of regular, well shaped, few micrometer crystals. Photoluminescence spectra of these crystals are characterized by fixed positions of very narrow exciton lines (FWHM down to $1 \mathrm{meV}$ ), where free excitons A, B, C, resolved two donor bound excitons and acceptor bound exciton are visible. Fixed position of exciton lines is in contrast to small changes of line energies which have been always observed for epitaxial GaN layers because of strain present in them. Free electron concentration of AMMONO GaN is less than few times $10^{15} \mathrm{~cm}^{-3}$, as estimated from EPR signal of shallow donor. The above-mentioned facts qualified these crystals as state of the art strain-free, model material for basic parameter measurements of $\mathrm{GaN}$. In this work, results of PL and EPR measurements performed on AMMONO GaN crystals are presented and discussed.

PACS numbers: 81.10.Dn

*This work was supported by the Committee for Scientific Research grants no. 8 T11 B 03310 and 7 T08 A 05110. 


\section{Introduction}

An enormous amount of papers published currently on $\mathrm{GaN}$ have been performed on strained material. Heteroepitaxial layers are always strained because of lattice mismatch and difference in thermal expansion coefficient between substrate and layer materials. Indications of small strain presence in homoepitaxial layers grown on high nitrogen pressure (HNP) [1] substrates have been also found.

Because of built-in strain, basic parameters of GaN cannot be determined exactly and the values of them (e.g. exciton line energies) change from paper to paper. Although some authors believe that they have unstrained samples, the controversy about the values of basic GaN parameters is still an alive issue.

In this paper we present results of experiments performed on GaN crystals obtained by AMMONO method. These crystals grow spontaneously from ammonia solution in form of regular, well shaped, few micrometers grains where none of crystallographic directions is distinguished. The observed energy positions of exciton photoluminescence lines are not sample dependent. Such facts qualified AMMONO crystals as state of the art strain-free, model material for measurements of basic parameters of $\mathrm{GaN}$.

\section{AMMONO GaN crystals}

The samples were obtained by AMMONO method described elsewhere [2]. The AMMONO technique allowed to grow nitride crystals at relatively low temperature and pressure conditions (our crystals were obtained at temperatures not exceeding $550^{\circ} \mathrm{C}$ and pressures below $5 \mathrm{kbar}$ ). Several growth processes were performed in the presence of rare-earth elements, applied in metallic form. An autoclave was loaded in pure nitrogen atmosphere, evacuated down to $10^{-2} \mathrm{mbar}$, and then filled with ammonia. Following the growth processes, $\mathrm{GaN}$ crystals were treated with aqua regia and rinsed in distilled water. Depending on the growth conditions, the crystals had either a form of regular grains of a few micrometer size or were of a needle shape up to $25 \mu \mathrm{m}$ length. X-ray diffraction pattern showed that the phase of AMMONO GaN microcrystals was purely hexagonal (wurtzite structure). The preliminary results of lattice parameter measurements by Laue diffraction method were $a=3.1885(2) \AA$ and $c=5.1842(4) \AA$.

\section{Optical properties of AMMONO GaN}

All GaN crystals obtained by AMMONO method revealed very intensive and homogeneous photoluminescence (PL), also at room temperature. The intensity of the observed PL signal was a few times higher than for the best homoepitaxial and heteroepitaxial GaN layers. Some examples of PL spectra of undoped AMMONO GaN are shown in Fig. 1. For such samples only commonly known "yellow" wide band, covering the whole visible region in the range of 1.5 to $2.9 \mathrm{eV}$ is present. The shape of this band varied significantly from sample to sample, depending on the growth conditions. Analysis of a large number of spectra (some of them presented in Fig. 1) allowed to recognize this band as a superposition of several peaks and attribute its shape changes to different intensities of constituent peaks.

The "yellow" band is commonly ascribed to electron recombination at a deep level strongly coupled to the lattice [3]. Our results showed that a whole family of 


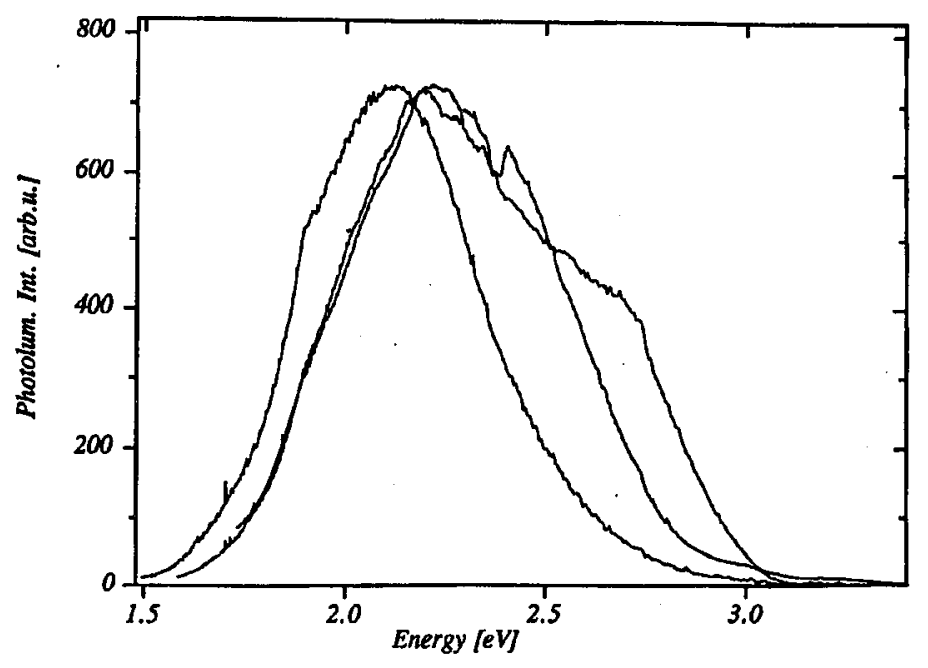

Fig. 1. Photoluminescence spectra (measured at $4.2 \mathrm{~K}$ ) of GaN crystals obtained by AMMONO method without the presence of rare-earth elements.

such levels exists in the middle of GaN band gap. However, the origin of this deep defect is still an open issue. Analogical bands found for other semiconductors (i.e. $\mathrm{GaAs})$ have been connected to metallic vacancy $\left(\mathrm{V}_{\mathrm{Ga}}\right)$ or metallic vacancy based complex ( $\mathrm{V}_{\mathrm{Ga}}$-shallow donor) [4]. On the other hand, for similar wide PL bands observed for AlN samples, some correlation of band shape and position with oxygen content have been found [5]. Consequently, complexes involving oxygen (most probably with aluminum vacancy) have been proposed as an origin of "yellow" band in AlN. Therefore, gallium vacancy and/or oxygen are strong candidates for deep defect causing "yellow" luminescence of GaN.

Typical PL spectrum of GaN crystals grown in the presence of rare-earth elements (RE) is shown in Fig. 2. A dramatic improvement of PL properties, in a sense of decrease of "yellow" band intensity and increase of strong and sharp structures due to exciton recombinations was characteristic for all AMMONO GaN:RE samples. Exciton region of GaN:RE PL spectrum (see insert of Fig. 2), was very similar to the spectra observed for the best homoepitaxial GaN layers. The FWHM of the exciton lines reached the record values of $1 \mathrm{meV}$. What should be stressed, the energy positions of exciton lines were not sample dependent, in contrast to small changes always seen even for homoepitaxial GaN layers and attributed to strain present in them. This experimental observation proved strain-free structure of AMMONO GaN crystals. Therefore, energy position of bound excitons $E_{\mathrm{XD}}=3.4719 \mathrm{eV}$ and $E_{\mathrm{XA}}=3.4664 \mathrm{eV}$ determined for them could be used as a reference data for GaN crystals grown by other techniques.

The intensity of XA line was typically higher than the intensity of XD line in AMMONO GaN:RE. This observation together with the narrow structure of exciton lines suggested low electron concentration in the GaN grains. Extremely small width of excitonic lines allowed to distinguish two donor bound excitons. 


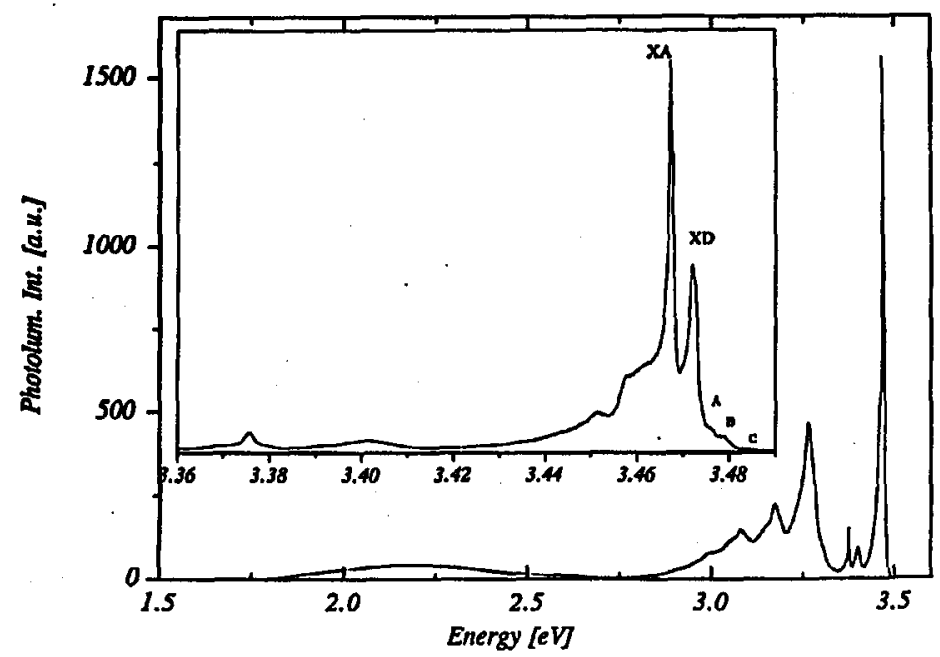

Fig. 2. PL spectra of AMMONO GaN grown in the presence of rare-earth elements. Magnified exciton region is shown in the insert.

Besides bound exciton lines there were free exciton transitions seen in the ultraviolet luminescence spectra. Three free excitons A, B and C were observed (Fig. 2). They were due to the split of GaN valence band to subbands of $\Gamma_{9}, \Gamma_{7}$ and $\Gamma_{7}$ symmetries, respectively.

\section{EPR properties of AMMONO GaN}

EPR measurements revealed two resonance absorption lines (Fig. 3) with $g=$ 1.9494 and $g=2.0029$. Since the experiments were performed on microcrystalline powder, no orientation dependence of resonances could be followed. The line of $g=1.9494$ and $7.5 \mathrm{mT}$ width was observed in most of the AMMONO crystals and identified as an average signal of an anisotropic line seen for heteroepitaxial undoped (n-type) GaN layers and attributed to an effective mass donor [6]. The second resonance with $g=2.0029$ appeared in all AMMONO crystals grown in the absence of rare earths and was analogical to the signal observed for $\mathrm{GaN}$ layers grown by metal organic chemical vapor deposition (MOCVD) epitaxy [7]. We would like tentatively to attribute this signal to oxygen impurity. The main reason for this identification is a lack of this resonance for crystals grown in the presence of rare earths, which are known to getter oxygen very efficiently. A simultaneous measurement of AMMONO GaN:RE crystals and $\mathrm{Si}: \mathrm{Fe}$ standard sample connected with the precise weighting of these two samples allowed us to estimate the spin concentration in AMMONO GaN as about $5 \times 10^{15} \mathrm{~cm}^{-3}$ centers. This is much lower than in any uncompensated $\mathrm{GaN}$ grown by other techniques.

\section{Summary}

The results of PL and EPR measurements performed on AMMONO GaN were presented. These strain-free crystals allowed to obtain the correct values of bound exciton energies for GaN. Knowledge of these parameters for pure GaN 


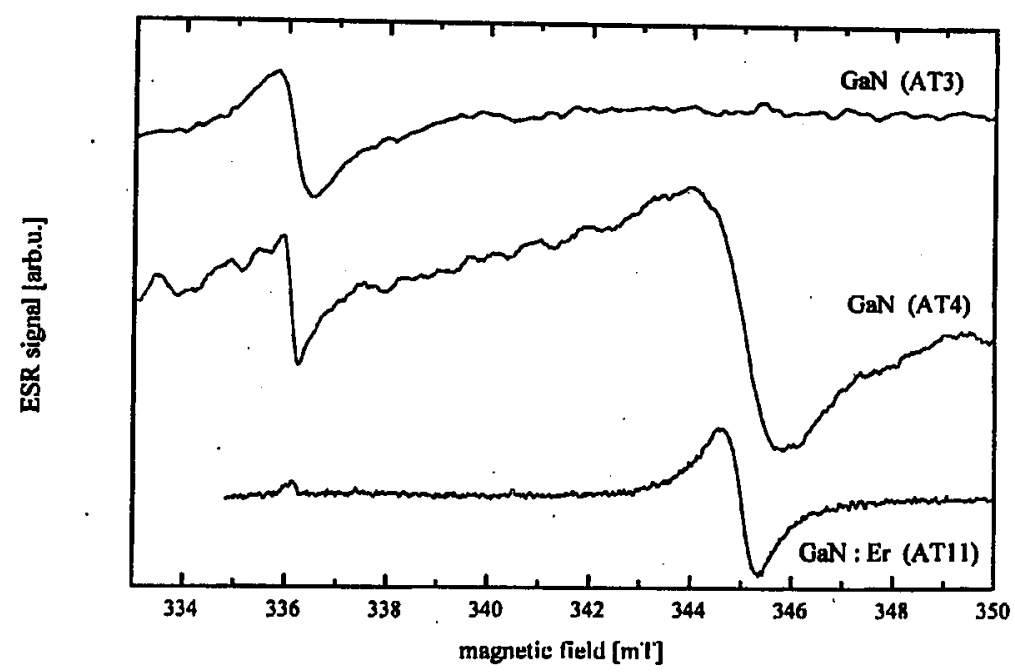

Fig. 3. EPR spectra of AMMONO GaN grown in the presence of small (AT3) and high (AT4) amount of lithium mineralizer. Annihilation of the resonance line at lower magnetic field and narrowing of the resonance line at higher magnetic field caused by rare-earth elements present during growth process is visible (AT11).

(wurtzite structure) will be essential for construction and performance of short wavelength lasers and other optoelectronic devices. A new EPR signal with $g=2.0029$ was observed in crystals grown in the absence of rare earths and was tentatively attributed to oxygen impurity or gallium vacancy.

\section{Acknowledgments}

We acknowledge Prof. M. Lefeld-Sosnowska and E. Leśkiewicz for X-ray measurements.

\section{References}

[1] S. Porowski, J. Jun, M. Boćkowski, M. Leszczyniski, St. Krukowski, M. Wróblewski, B. Lucznik, I. Grzegory, in: Proc. 8th Conf. on Semi-insulating III-V Materials, Warsaw 1994, Ed. M. Godlewski, World Scientific, Singapore 1994, p. 61.

[2] R. Dwiliński, J.M. Baranowski, M. Kamińska, R. Doradziński, J. Garczyński, L. Sierzputowski, H. Jacobs, Acta Phys. Pol. A 90, 763 (1996).

[3] J. Neugebauer, C.G. Van de Walle, Appl. Phys. Lett. 69, 503 (1996).

[4] E.W. Williams, Phys. Rev. 168, 922 (1968).

[5] S. Pačesová, L. Jastrabík, Phys. Status Solidi B 93, K111 (1979).

[6] W.E. Carlos, J.A. Freitas, M. Asif Khan, D.T. Olson, J.N. Kuznia, Phys. Rev. B 48, 17878 (1993).

[7] B. Suchanek, M. Palczewska, K. Pakuła, J. Baranowski, M. Kamińska, Acta Phys. Pol. A 92 (1997), Proc. of this Conf. (Part II). 